\title{
Mobile Robot Self-localization Based on Multi-sensor Fusion Using Limited Memory Kalman Filter with Exponential Fading Factor
}

\author{
Xueli Cheng ${ }^{1 *}$, Wanli Liu' ${ }^{2}$, Meng Guo ${ }^{2}$ and Zhenhua Zhang ${ }^{3}$ \\ ${ }^{1}$ Department of Mechanical Engineering, Henan Institute of Technology, Xinxiang 453002, China \\ ${ }^{2}$ School of Mechatronics Engineering, China University of Mining and Technology, Xuzhou 221116, China \\ ${ }^{3}$ School of Mechanical and Civil Engineering, Curtin University, Perth, Bentley 6102, Australia
}

Received 2 October 2018; Accepted 27 December 2018

\begin{abstract}
Accumulative errors can be retained all the time when classical Kalman filtering is adopted for odometer-based dead reckoning, thereby affecting self-localization accuracy of the robot. A mobile robot self-localization method based on limited memory Kalman filtering (LMKF) with exponential fading factor was proposed to reduce accumulative errors of the odometer and improve localization accuracy of the mobile robot. The self-localization system of mobile robot was built. A mathematical model was established based on LMKF with exponential fading factor. A dead reckoning method fusing multi-sensor information was proposed. The model accuracy was verified through simulation and test. Results indicate that the LMKF method with exponential fading factor positively affects the tracking of high-speed maneuvering dynamic targets, and its localization error is reduced by $42.5 \%$ compared with the odometer-based dead reckoning. The tracking accuracy of the mobile robot is stable at $0.5 \mathrm{~m}$. This study can provide references for mobile robot selflocalization using multi-sensor.
\end{abstract}

Keywords: Mobile robot, Self-localization, Multi-sensor Information Fusion, LMKF with Exponential Fading Factor

\section{Introduction}

The self-localization system acquires position, posture, velocity, and external environmental information of the mobile robot through multiple sensors to realize its real time, reliable, and accurate localization, it is one of the key technologies of mobile robots. In recent years, with the rapid development of mobile robot technology, the application requirements of self-localization systems have also presented gradual growth trend. Relevant technical studies have achieved great progress, and increasing number of units has participated in the study, design, production and application of the mobile robot self-localization system. The traditional self-localization system uses a single sensor to acquire information with large errors and uncertainties. Mobile robots cannot easily adapt to the complex environment. Therefore, multi-sensor data acquisition is a reliable scheme for mobile robot self-localization. Application of the multi-sensor information fusion technology to mobile robots can provide accurate decisions on the behavior of robots and improve the accuracy of mobile robot self-localization. Thus, the combination of mobile robot and the multi-sensor information fusion technology has become a research hotspot. Information fusion technology is widely used in the mobile robot selflocalization. Multi-sensor information is integrated based on fuzzy logic algorithm. The collision-free mobile robot navigation system is designed, and intelligent obstacle avoidance of mobile robots is successfully realized [1]. In addition, the multi-information fusion technology based on

*E-mail address: chengxueli2005@126.com

ISSN: $1791-2377$ @ 2018 Eastern Macedonia and Thrace Institute of Technology. All rights reserved. doi:10.25103/jestr.116.24 wireless sensor network [2] has also improved selflocalization accuracy of mobile robot.

However, with the high intelligence of mobile robots, the pose information required by robots become increasingly complex, and uncertain and external interference factors are increasingly found. Effectively processing multi-sensor measurement data in real time has become a key issue to achieve multi-sensor information fusion. To solve this problem, improve the system localization accuracy, and eliminate the information fusion cumulative errors, a multisensor data fusion algorithm based on the physical model is generally adopted to eliminate or reduce interference signals and extract useful signals. The most effective among the methods is the Kalman filtering algorithm. When the number of measurements continuously increases in the classical Kalman filtering process, the mean square error of state estimation tends to be stable, but the deviation of the state estimation value from the actual value tends to increase, and the filter divergence phenomenon appears, greatly challenging the study of self-localization systems.

Scholars conducted substantial studies on the information fusion Kalman filter algorithm in the mobile robot self-localization system [3-4]. However, the system mathematical model and noise model of the information fusion are extremely coarse or distorted. Thus, the actual physical process of the self-localization system cannot be reflected. Therefore, establishing a Kalman filter fusion algorithm with accurate mathematical model can accurately fuse multi-sensor information in real time and then accurately calculate the stable and reliable track of mobile robots, an urgent problem to be solved.

A mathematical model of limited memory Kalman filtering (LMKF) with exponential fading factor was 
established by improving the LMKF, and the multi-sensor information of digital gyroscope, odometer, and other sensors were fused into the dead reckoning method to improve the mobile robot self-localization accuracy, thereby providing a reference for the development and optimization of mobile robot.

\section{State of the art}

At present, studies on information fusion algorithms in the mobile robot self-localization system have been performed. Klančar [3] used extended Kalman filter to carry out synchronous localization and mapping (SLAM) of mobile robots and improved the SLAM algorithm convergence estimated by the noise covariance matrix. However, he failed to analyze the influence of prediction mean square error on the algorithm. Alatise [4] designed an extended Kalman filter, which combines inertia data and visual data to amend each sensor linkage, and acquired accurate direction and position of the mobile robot. In addition, his method is unsuitable in a broad environment. Simanek [5] designed a multi-mode data fusion algorithm based on the extended Kalman filter. The algorithm could accurately identify abnormal data and realize standard statistical test of filter residual, but the influence of the filter gain matrix on the algorithm was neglected. Lee [6] introduced an algorithm detecting and eliminating magnetic disturbance in the geomagnetic field. The algorithm was implemented in a mini type microprocessor and sensor module with relatively low costs by combining the extended Kalman filter, but it is unsuitable for robot localization in the area without magnetic disturbance. Jiang [7] put forward a new kinematical calibration method based on extended Kalman filter and particle filter algorithms. This method could remarkably improve the localization accuracy of the robot, but did not involve the influence of fading factors on filtering. $\mathrm{Yu}$ [8] proposed a generalized full Kalman filter algorithm based on Gaussian-Newton method, which could process all random errors in various equations of the nonlinear dynamic errorsin-variables model, and improved the state estimation errors. However, the prediction mean square error was not studied. Gualda [9] studied the application of the extended Kalman filter in indoor navigation of mobile robot, combined the relative localization obtained by robot odometer measurement with the absolute localization measured by a group of ultrasonic local localization systems, but did not analyze the influence on estimated mean square error. Qian [10] fused data of Kinect visual sensor and four wheel-type encoders, used the extended Kalman filter with specific processing to localize the mobile robot, but did not further study the dead reckoning method after the fusion. Pak [11] proposed a new intelligent filtering algorithm, namely, selfhealing extended Kalman filter, which was only restricted to fault diagnosis. Luo [12] put forward a multi-information fusion model applied to the localization and navigation system of the wheel-type mobile robot. Mr Luo used Kalman filtering to realize data layer fusion of quantitative information of the system and artificial neural network to realize decision-making layer fusion of qualitative information of the system. However, separating quantitative information from qualitative information was ineffective in information integration. Zhang [13] put forward a navigation control method based on multi-sensor information fusion of charge-coupled device imaging sensor, electronic compass, accelerometer, and ultrasonic, which realized navigation control of car path tracking. However, only the sensor information in front of the car were fused. Zhang [14] fused ultrasonic sensor information, laser ranging information, and map information through particle filtering to realize accurate dynamic tracking and localization of indoor robots. However, this method was inapplicable to outdoor robots. Li [15] fused analog input data acquired by sensors and conversion data displayed on the upper computer into the improved particle swarm filter localization model. The model improved the localization accuracy of mobile robots indoors by a large margin, but only front-end sensor information of robot was acquired and fused. Mao [16] used particle filtering dynamic tracking and localization method to realize the self-localization of the microrobot, but it was only suitable for places with map information. Wang [17] used the extended Kalman filter algorithm to fuse binocular stereoscopic vision and odometer information, and realized real time localization of mobile robot's travel direction. However, directional angle was not involved. Qin [18] used the traditional Kalman filtering to fuse measurement data by odometer and ultrasonic sensor, amended the positions of mobile robots during motion, but did not consider the filtering divergence problem. Xie [19] introduced the driving force of mobile robots and the frictional force with the ground as correction factor for prediction errors of the filtering algorithm. Mr Xie combined the input of multimodel with output of filtering results during the non-uniform motion process to form multi-mode Kalman filter. This filtering method greatly improved the real time tracking performance of non-uniform motion of mobile robots, but the influences of dynamic noise and observation noise model on the filtering performance were not taken into account. Gao [20] proposed a method, which used estimated mean square error and innovation covariance estimator to calculate multiple fading factors, which were introduced to realize weighed correction to each channel. Thus, the overall performance of the adaptive Kalman filter was improved, but the channel information was not fused. Xue [21] designed a new adaptive Kalman filtering with multiple fading factors, calculated innovation covariance estimator based on fading memory exponential weights and introduced multiple fading factors to adjust the prediction error covariance matrix. Therefore, each filtering channel had different adjustment capabilities, which improved the precision and robustness of the filtering algorithm. However, the estimated mean square error was not analyzed.

A variety of information fusion algorithms for robot's self-localization were proposed based on this analysis, thereby improving the accuracy and adaptability of the mobile robot's self-localization to some degree. However, few algorithms have considered using the LMKF method with exponential fading factor to control multi-sensor fusion errors and the work on reducing cumulative error of localization by combining attenuation memory filter and limited memory filter. LMKF with exponential fading factor was designed. This filter was used to fuse multi-sensor information into the dead reckoning method, and the simulation analysis and experimental study on the localization method after information fusion were carried out. The effectiveness of this self-localization method was verified.

The remainder of this study is organized as follows. Section 3 constructs an LMKF physical model with exponential fading factor based on the LMKF, and proposes a dead reckoning method fusing multi-sensor information. Section 4 conducts a simulation analysis of the LMKF with 
exponential fading factor, and analyzes the experimental results of dead reckoning based on multi-sensor information fusion. Section 5 summarizes the conclusions.

\section{Methodology}

\subsection{Self-localization system of mobile robot}

The self-localization system of mobile robot consists of two components, namely, hardware and software.

The hardware part, which is the foundation of realizing self-localization of mobile robot, consists of localization sensors. A single sensor can only acquire local information of the environment or measured object, but multi-sensor can comprehensively restore features of the ambient environment or measured object and can enhance the robustness of the localization system. In the comprehensive comparison of typical sensor performances, HMC5883L 3D magnetic resistance-type electronic compass, ADXL345 three-axis acceleration sensor, L3G4200D three-axis digital gyroscope, and odometer (WTK-10 sensor) are selected as the hardware foundation of the self-localization system.

The software part uses the LMKF method with exponential fading factor as the control strategy of the system, as shown in Fig. 1. The hardware part collects the pose information and environmental information of mobile robots. The software part uses the geometric method and the LMKF method with exponential fading factor to realize fusion calculation, coordinate transformation on the information, reduce accumulative errors caused by dead reckoning, and reliably output position and pose information of mobile robots in real time.

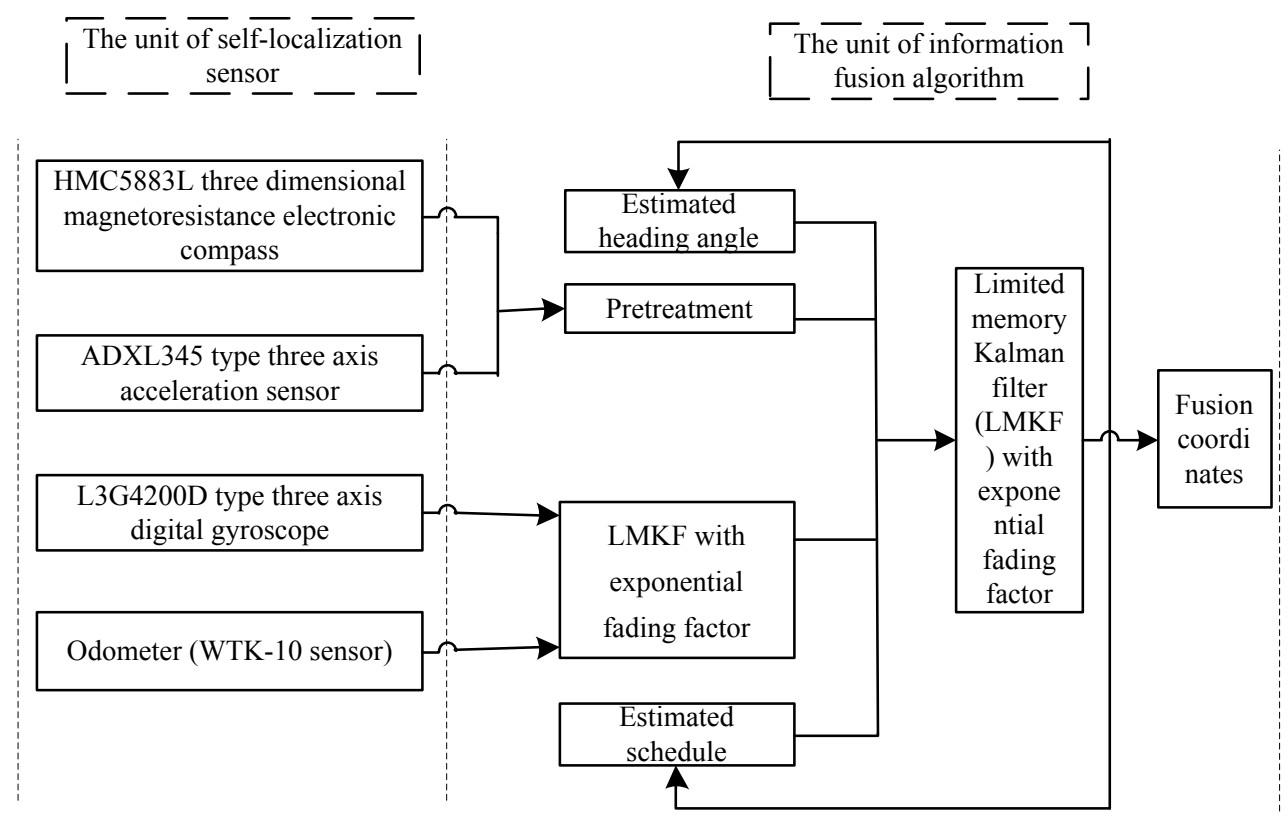

Fig. 1. Self-localization system of mobile robot

\subsection{Design of LMKF with exponential fading factor}

\subsubsection{Mathematical model}

The method using a single sensor to calculate robot poses divergently has poor accuracy. Thus, improving the selflocalization accuracy using a filtering algorithm is necessary. When the classical Kalman filtering is used for multi-sensor information fusion, the system mathematical model and noise model are extremely coarse or distorted. Thus, they cannot reflect the actual physical process of the selflocalization system, thereby generating filtering divergence phenomenon. The LMKF method with exponential fading factor is used to control fusion errors of multi-sensor.

The limited memory filter with exponential fading factor only retains measured values of the $N$ times closest to the time $k$ and abandons measured values before $k-N+1$ time [22], meanwhile, the exponential fading factor is introduced, further increasing the weight of the measured value close to the current moment, thereby combining the fading memory filtering and limited memory filter to reduce the cumulative error.

The derivation is as follows: $k$-time measurement is performed on $X_{k}$, and its previous discrete values and measured values are the following: $Z_{1}, Z_{2}, \mathrm{~L}, Z_{d}, \mathrm{~L}, Z_{k-1}, Z_{k}$. Assuming that $Z_{d}$ to $Z_{k}$ has a total of $\mathrm{N}+1$ measured value, it is recorded as follows:

$\bar{Z}_{d, k}^{\mathrm{N}+1}=\left[\begin{array}{c}Z_{d} \\ Z_{d+1} \\ \vdots \\ Z_{k-1} \\ Z_{k}\end{array}\right], \bar{Z}_{d, k-1}^{\mathrm{N}}=\left[\begin{array}{c}Z_{d} \\ Z_{d+1} \\ \vdots \\ Z_{k-1}\end{array}\right], \bar{Z}_{d+1, k}^{\mathrm{N}}=\left[\begin{array}{c}Z_{d+1} \\ \vdots \\ Z_{k-1} \\ Z_{k}\end{array}\right]$

$\hat{X}_{k}^{N+1}=E^{*}\left[X_{k} / \bar{Z}_{d, k}^{\mathrm{N}+1}\right]$

$\hat{X}_{k / k-1}^{N}=E^{*}\left[X_{k} / \bar{Z}_{d, k-1}^{\mathrm{N}}\right]$

$\hat{X}_{k}^{N}=E^{*}\left[X_{k} / \bar{Z}_{d+1, k}^{\mathrm{N}}\right]$

Meanwhile, the linear relation between $\hat{X}_{k}^{N+1}$ and $\hat{X}_{k}^{N}$ is established. Then, the linear relation between $\hat{X}_{k}^{N}$ and $\hat{X}_{k / k-1}^{N}$ is determined according to the two equations. In the 
meantime, scalar exponential fading factor $\lambda_{k}$ is introduced into the estimated mean square error in the mean square error equation of one-step prediction, and the limited memory filter equation with exponential fading factor of $X_{k}$ is finally obtained, as follows:

$$
\begin{aligned}
& \left\{\begin{array}{l}
\hat{X}_{k}^{N^{*}}=\Phi_{k, k-1} \hat{X}_{k-1}^{N^{*}}+K_{k}^{*}\left(Z_{k}-H_{k} \Phi_{k, k-1} \hat{X}_{k-1}^{N^{*}}\right), k<N \\
\hat{X}_{k}^{N^{*}}=\Phi_{k, k-1} \hat{X}_{k-1}^{N^{*}}+K_{k}^{*}\left(Z_{k}-H_{k} \Phi_{k, k-1} \hat{X}_{k-1}^{N^{*}}\right)-\bar{K}_{k}^{*}\left(Z_{d}-H_{d} \Phi_{d, k} \Phi_{k, k-1} \hat{X}_{k-1}^{N^{*}}\right), k>N
\end{array}\right. \\
& \left\{\begin{array}{l}
P_{k}^{N^{*}}=\left(I-K_{k}^{*} H_{k}\right) P_{k / k-1}^{N^{*}}\left(I-K_{k}^{*} H_{k}\right)^{T}+K_{k}^{*} R_{k}\left(K_{k}^{*}\right)^{T}, k<N \\
P_{k}^{N^{*}}=\left[\left(\Phi_{k, k-1} \lambda_{k} P_{k / k-1}^{N^{*}} \Phi_{k, k-1}^{T}+\Gamma_{k-1} Q_{k-1} \Gamma_{k-1}^{T}\right)^{-1}+H_{k}^{T} R_{k}^{-1} H_{k}-\Phi_{d, k}^{T} H_{d}^{T} R_{d}^{-1} H_{d} \Phi_{d, k}\right]^{-1}, k>N
\end{array}\right. \\
& K_{k}^{*}=P_{k}^{N^{*}} H_{k}^{T} R_{k}^{-1} \\
& \bar{K}_{k}^{*}=P_{k}^{N^{*}} \Phi_{d, k}^{T} H_{d}^{T} \bar{R}_{d}^{-1}, k>N
\end{aligned}
$$

In Equations (5)-(8), $\hat{X}_{k}^{N^{*}}$ is state estimation, $P_{k}^{N^{*}}$ is the estimated mean square error, $P_{k / k-1}^{N^{*}}$ is the mean square error of one-step prediction, $K_{k}^{*}$ and $\bar{K}_{k}^{*}$ are filter gain matrixes, $\lambda_{k}$ is the fading factor of fading memory filter and defined as exponential function, $\mathrm{N}$ is limited memory length, asterisk * represents the addition of an exponential fading factor.

When $k$ is smaller than or equal to memory length $N$ at the measuring time. The LMKF with exponential fading factor cannot be implemented, but the traditional fading memory Kalman filtering can be performed by calculating the initial values $\hat{X}_{0}=E\left[X_{0}\right]$ and $P_{0}=\operatorname{Var}\left[X_{0}\right]$ to $\hat{X}_{N}^{*}$ and $P_{N}^{*}$. However, in $k=N+1$, if $\hat{X}_{N}^{N^{*}}=\hat{X}_{N}^{*}$ is directly considered, $P_{N}^{N^{*}}=P_{N}^{*}$ will be considered as the initial value for limited memory filter, and then the follow-up filtering value $\hat{X}_{k}^{N^{*}}$ will always be affected by $\hat{X}_{0}$ and $P_{0}$, thereby not satisfying the requirements of limited memory filter. To eliminate the influence of initial values on the limited memory filter, the initial values are considered according to the following equations [23]:

$$
\begin{aligned}
& P_{N}^{N^{*}}=\left[\left(P_{N}^{*}\right)^{-1}-\Phi_{0, N}^{T}\left(\lambda_{0} P_{0}\right)^{-1} \Phi_{0, N}\right]^{-1} \\
& X_{N}^{N^{*}}=P_{N}^{N^{*}}\left[\left(P_{N}^{*}\right)^{-1} \hat{X}_{N}^{*}-\Phi_{0, N}^{T}\left(\lambda_{0} P_{0}\right)^{-1} \hat{X}_{0}\right]
\end{aligned}
$$

The variance matrix $Q_{k}$ of the system noise sequence and variance matrix $R_{k}$ of the measured noise sequence on the filtering are indirectly affected by the gain matrixes. Therefore, under the condition that the system equation is inaccurate or unknown, the filter gains $K_{k}^{*}$ and $\bar{K}_{k}^{*}$ can be directly estimated without estimating $Q_{k}$ and $R_{k}$. At the time, $\lambda_{k}$ has an important influence on the filtering. The exponential fading factor $\lambda_{k}$ is defined as follows:

$$
\lambda_{k}=\left\{\begin{array}{l}
1,\left(\alpha_{k}<1\right) \\
e^{\alpha_{k}-1},\left(\alpha_{k} \geq 1\right)
\end{array}\right.
$$

Fig. 2 shows the flowchart of the LMKF with exponential fading factor. When $a_{k}$ is equal to 1 , the obtained filtering result is the most ideal; when $a_{k}$ is greater or smaller than 1 , fading factor $\lambda_{k}$ is determined by the exponential function with $e$ as the base, which indirectly affects the gain matrixes $K_{k}^{*}$ and $\bar{K}_{k}^{*}$, and improvs the inaccuracy of system modeling and the unknown influence of noise characteristics on the filtering, and increasing the system's flexibility and robustness.

\subsubsection{Simulation experiment}

Simulation 1: $\alpha-\beta-\gamma$ filtering method [24] is used. The carrier moves along one straight line, and its initial position coordinates, initial velocity, and initial acceleration are $s_{0}=5 m, v_{0}=0 m / s, a_{0}=0.1 \mathrm{~m} / \mathrm{s}^{2}$, respectively. The initial state of the carrier is $\hat{X}_{0}=5 m, P_{0}=\left(\begin{array}{ccc}10 & 0 & 0 \\ 0 & 10 & 0 \\ 0 & 0 & 10\end{array}\right)$. The displacement is measured once every $1 \mathrm{~s}$, as the observed value of the Kalman filter. The Kalman filter assumes that the carrier has no initial velocity (i.e., $v_{0}=0 \mathrm{~m} / \mathrm{s}$ ) but moves at an initial acceleration of $a_{0}=0.1 \mathrm{~m} / \mathrm{s}^{2}$. Therefore, the equation of the estimated information is $\left\{\begin{array}{l}s_{k}=s_{k-1}+\frac{1}{2} a_{k-1} \\ v_{k}=v_{k-1}+a_{k-1} \\ a_{k}=a_{k-1}+j_{k-1}\end{array}\right.$, In the acceleration formula, $j_{k}$ is a random quantity for the observer and is described by white noise. $X_{k}=\left(\begin{array}{c}s_{k} \\ v_{k} \\ a_{k}\end{array}\right)$ is considered, and the state equation is $X_{k}=\Phi X_{k-1}+\Gamma j_{k-1}$.

In the equation, one-step transfer matrix is $\Phi=\left(\begin{array}{ccc}1 & 1 & 1 / 2 \\ 0 & 1 & 1 \\ 0 & 0 & 1\end{array}\right)$, and system noise drive matrix is $\Gamma=\left(\begin{array}{l}0 \\ 0 \\ 1\end{array}\right) . \quad Z_{k}=s_{k}+V_{k}=\left(\begin{array}{lll}1 & 0 & 0\end{array}\right)\left(\begin{array}{l}s_{k} \\ v_{k} \\ a_{k}\end{array}\right)+V_{k}$. Thus, the measuring matrix is $H=\left(\begin{array}{lll}1 & 0 & 0\end{array}\right)$. 
Due to the inaccuracy of the system equations, when the classical Kalman filter is adopted, the state noise and

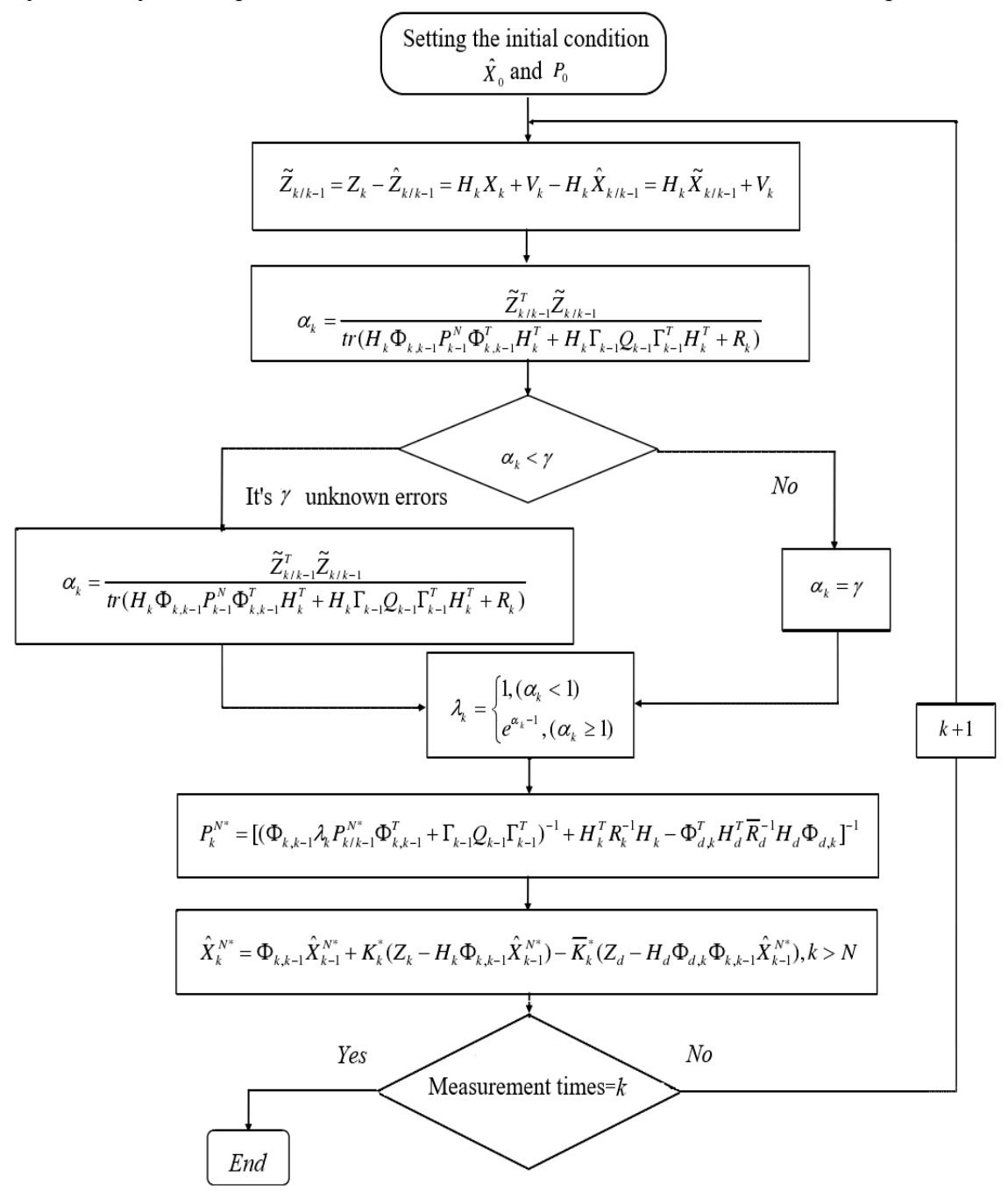

Fig. 2. Program flowchart of the LMKF with exponential fading factor

measured noise will cause the filter to diverge. To facilitate the comparison of the LMKF and the LMKF with exponential fading factor, each filter curve is enlarged.

Simulation 2: assuming that a two-coordinate radar observes a moving target on the plane, the target is under uniform linear motion along the $y$ axis at $t=0-400 \mathrm{~s}$, and the motion velocity and initial coordinates of the target are $15 \mathrm{~m} / \mathrm{s}$ and $(2000,10000)$, respectively. The target highspeed maneuvering at $t=400 \mathrm{~s}$ has an initial velocity of $10 \mathrm{~m} / \mathrm{s}$ and an acceleration of $24.975 \mathrm{~m} / \mathrm{s}^{2}$ along the direction of the $x$ axis. At $t=430 \mathrm{~s}$, the acceleration in the direction of $x$ axis turns into $0.975 \mathrm{~m} / \mathrm{s}^{2}$ until $t=530 \mathrm{~s}$. Subsequently, the acceleration declines to zero, and the target maintains a uniform motion until the observation ends. The observation period is $T=1 s$. The observation is carried out independently along the $x$ and $y$ axes, and the standard deviations of observed noises are $100 \mathrm{~m}$.

\subsection{Dead reckoning method based on multi-sensor information fusion}

\subsubsection{Dead reckoning based on odometer}

Dead reckoning principle based on odometer is shown in Fig.3. The robot is assumed to move on a $2 \mathrm{D}$ plane, and its coordinates at time $\mathrm{t}$ are $(x, y, \theta) . x$ and $y$ are the coordinate values of the robot on the rectangular coordinate system, and $\theta$ is the heading angle of the robot. The time interval $\Delta t$ is the period for the odometer count and the heading angle reading, $v_{L}$ and $v_{R}$ are the traveling linear velocities of the left and right driving wheels, respectively.

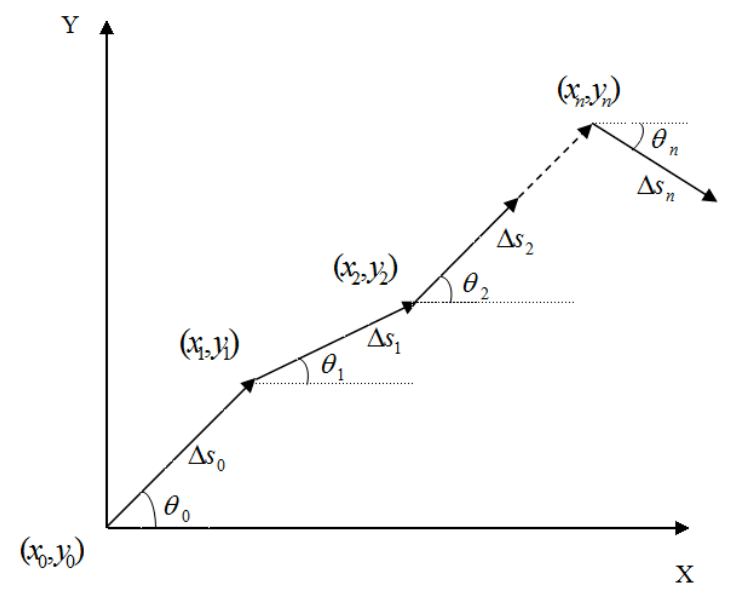

Fig. 3 Principle diagram of dead reckoning based on odometer

The arc tracks of left and right driving wheels within one period can be approximated as a segment of straight line because the sampling frequency of a single-chip microcomputer is usually very high. The length of this straight line 
is equal to the radius of the arc multiplied by the corresponding angle of the center of the circle. The displacement increment of the robot obtained by the dead reckoning is as follows:

$$
\left\{\begin{array}{l}
v=\frac{v_{R}+v_{L}}{2} \\
\omega_{d}=\frac{v_{R}-v_{L}}{t_{r}}
\end{array}\right.
$$

where $t_{r}$ represents the two-wheel spacing; $v$ and $\omega_{d}$ are the velocity and angular velocity of heading change measured by the odometer, respectively.

The coordinate reckoning formula of the mobile robot can be obtained as follows:

$$
\left\{\begin{array}{l}
x_{k+1}=x_{k}+v \cos \left(\theta_{k+1}\right) \Delta t \\
y_{k+1}=y_{k}+v \sin \left(\theta_{k+1}\right) \Delta t \\
\theta_{k+1}=\theta_{k}+\omega_{d} \Delta t
\end{array}\right.
$$

where $x_{k}$ and $y_{k}$ are the coordinate positions of the robot center. $\theta_{k}$ is the heading angle of the robot, indicating the angle between the heading of the robot and the positive direction of $x$ axis of the coordinate system. It can be converted from the angle of the actual north direction measured by the electronic compass. The method that calculates the position and pose of the robot through the above formula is called odometer dead reckoning algorithm.

Theoretically, the odometer dead reckoning algorithm can calculate the arbitrary length walking path of the robot, but each reckoning has an error because the straight line is used instead of the arc in the reckoning process. Moreover, the coded disc and odometer itself have systematic errors. Thus, the error of the dead reckoning presents infinite accumulation trend as the robot stroke increases. Therefore, relying solely on odometer and dead reckoning cannot estimate the pose of the mobile robot, and optimizing the dead reckoning method is necessary.

\subsubsection{Dead reckoning based on multi-sensor information fusion}

The accuracy of the robot pose cannot be estimated effectively only by relying on single sensors. To improve the self-localization accuracy, improved Kalman filtering algorithm is used to fuse the odometer and gyroscope data, and the state variable is considered as follows [25]:

$$
X=\left[\begin{array}{lll}
v_{L} & v_{R} & \omega_{g}
\end{array}\right]^{T}
$$

where $\omega_{g}$ is the angular velocity of the gyroscope, and the state equation of the system is as follows:

$$
X_{k}=\Phi_{k, k-1} X_{k-1}+\Gamma_{k-1} W_{k-1}
$$

where $\Phi_{k, k-1}$ is the one-step state transition matrix from time $t_{k-1}$ to time $t_{k}$. The expression is shown in Equation (16), where $t_{\mathrm{r}}$ is the two-wheel spacing; $W$ is the system noise matrix with covariance of $Q ; \Gamma_{k-1}$ is the driving matrix of the system noise matrix and the unit matrix $I$ in this system.
$\Phi_{k, k-1}=\left[\begin{array}{ccc}1 & 0 & 0 \\ 0 & 1 & 0 \\ \frac{-1}{t r} & \frac{1}{t r} & 0\end{array}\right]$

The measuring equation of the system is as follows:

$Z_{k}=H_{k} X_{k}+V_{k}$

where $H_{k}$ is the system measurement matrix and the unit matrix $I$ in the system; $V_{k}$ is the measurement noise matrix with covariance of $R$.

These parameters are brought into the LMKF with exponential fading factor Equations (5)-(8). The estimated values of the system variables are calculated, and the estimated values are substituted into Equations (12) and (13). The reckoning value of odometer after fusion of the gyroscope and the odometer can be obtained. The reckoning values of the odometer refer to dead reckoning values after the fusion.

The cumulative error of the three-axis digital gyroscope gradually increases with time, which reduces the reckoning accuracy of the odometer. This study uses the 3D magnetoresistive electronic compass to compensate the dead reckoning value of odometer. The selected $3 \mathrm{D}$ electronic compass has high measurement accuracy. Prior to fusion, the data of the electronic compass should be pretreated with time and coordinate system alignment. A three-axis accelerometer should be used for inclination compensation. The heading angle refers to the heading angles after fusion. The system state variable is obtained as follows:

$$
X=\left[\begin{array}{lll}
\theta & \omega_{d} & a_{\omega}
\end{array}\right]^{T}
$$

where $\theta$ is the heading angle of the robot; $a_{\omega}$ is the angular acceleration of the heading angle change of the robot.

The rotational motion of the motor can be the uniform angular velocity motion because the mobile robot moves slowly, and the state variable of the system is simplified as follows:

$$
X=\left[\begin{array}{ll}
\theta & \omega_{d}
\end{array}\right]^{T}
$$

The state equation of the system is as follows:

$X_{k}=\Phi_{k, k-1} X_{k-1}+\Gamma_{k-1} W_{k-1}$

where $\Phi_{k, k-1}=\left[\begin{array}{ll}1 & T \\ 0 & 1\end{array}\right]$, and $T$ is the time interval of the system sampling. The measuring equation of the system is as follows:

$$
Z_{k}=H_{k} X_{k}+V_{k}
$$

Similarly, these parameters are substituted into the LMKF with exponential fading factor Equations (5)-(8) to calculate the heading estimate. Then, the heading estimate is used to compensate for the cumulative errors of the odometer heading, and the dead reckoning simulation result 
by using the limited memory filtering method with exponential fading factor is obtained.

The simulation results obtained by the odometer-based dead reckoning method and the limited memory filtering reckoning algorithm with exponential fading factor are shown in Fig. 4. Fig. 4(a) shows the actual track curve of the mobile robot, estimated track of the odometer dead reckoning, and the estimated track of the filter dead

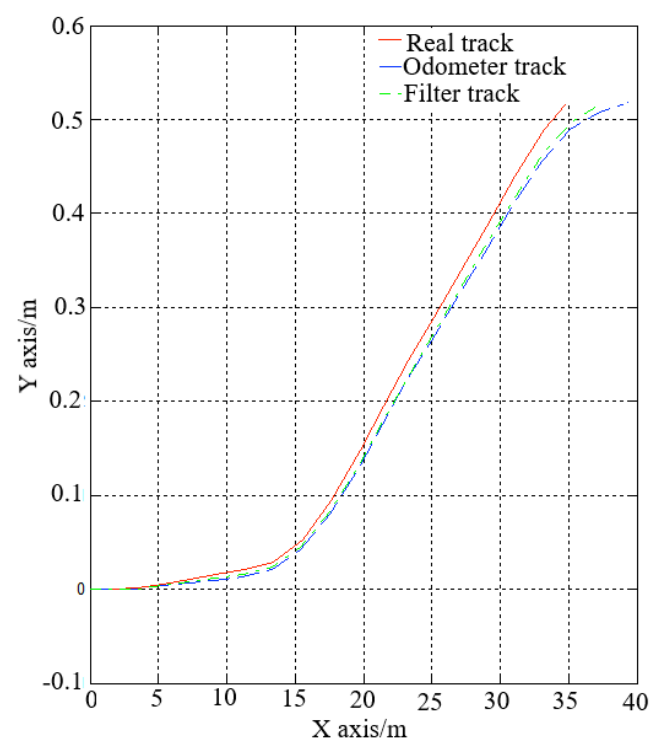

(a) Track curves

Fig. 4 Filtering method-based dead reckoning simulation diagram

\subsubsection{Track test with multi-sensor information fusion}

The initial position of the mobile robot is selected as the coordinate origin. The telerobot moves along the track $a-b 0$ $c 0-d 0-e 0-f 0-g 0-h 0$, which is the actual track of the mobile robot. $a-b 1-c 1-d 1-e 1-f 1-g 1-h 1$ is the track reckoned by the mobile robot using electronic compass and odometer; $a-b 2$ $c 2-d 2-e 2-f 2-g 2-h 2$ is the mobile robot track obtained by the limited memory filtering with exponential fading factor and multi-sensor information fusion method. The test comparison of odometer dead reckoning tracks before and after fusion is carried out, and the coordinate values of all tracks are obtained, as shown in Table 1.

Table. 1. Coordinate values of actual track, odometer track, and filter track

\begin{tabular}{l|l|l|l|l|l}
\hline \multicolumn{2}{c}{ Real track } & \multicolumn{2}{c|}{ Odometer track } & \multicolumn{2}{c}{ Filter track } \\
\hline $\begin{array}{l}\text { Coor } \\
\text { di- } \\
\text { nate } \\
\text { point }\end{array}$ & $\begin{array}{l}\text { Coordi- } \\
\text { nate } \\
\text { value }\end{array}$ & $\begin{array}{l}\text { Coord } \\
\text { i-nate } \\
\text { point }\end{array}$ & $\begin{array}{l}\text { Coordi- } \\
\text { nate value }\end{array}$ & $\begin{array}{l}\text { Coor } \\
\text { di- } \\
\text { nate } \\
\text { point }\end{array}$ & $\begin{array}{l}\text { Coordi- } \\
\text { nate value }\end{array}$ \\
\hline$a$ & 0,0 & $a$ & 0,0 & $a$ & 0,0 \\
$b 0$ & $5.4,0$ & $b 1$ & $3.87,0.03$ & $b 2$ & $4.17,0.04$ \\
$c 0$ & $7.2,0$ & $c 1$ & $5.34,0.02$ & $c 3$ & $6.54,0.02$ \\
$d 0$ & 9,0 & $d 1$ & $6.42,-0.02$ & $d 2$ & $8.58,-0.03$ \\
$e 0$ & $9,-1.2$ & $e 1$ & $6.16,-0.99$ & $e 2$ & $7.81,-1.25$ \\
$f 0$ & $3.6,-1.2$ & $f 1$ & $2.08,-1.33$ & $f 2$ & $1.96,-1.25$ \\
$g 0$ & $1.8,-1.2$ & $g 1$ & $2.33,-1.27$ & $g 2$ & $2.22,-1.21$ \\
$h 0$ & $0,-1.2$ & $h 1$ & $-1.56,-1.2$ & $h 2$ & $-1.52,-1.17$ \\
\hline
\end{tabular}

\section{Result Analysis and Discussion}

4.1 Simulation result analysis of the LMKF with exponential fading factor

Using the Simulation 1 method stated in 3.2.2, the filter curves and error curves obtained are shown in Fig. 5 and 6, respectively. reckoning. Fig. 4(b) shows the localization error curves of the odometer dead reckoning and filter dead reckoning during the simulation, thereby indicating that errors continuously increase. Fig. 4 shows that the error obtained by the optimized filtering dead reckoning method is smaller than that by the odometer dead reckoning error, and the minimization control of the odometer error is realized.

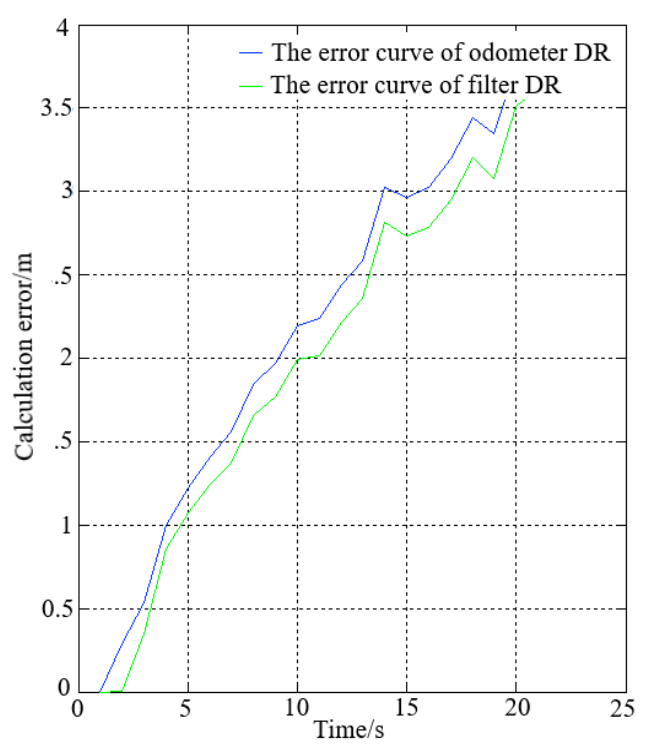

(b) Localization error curves

Fig. 5 and 6 show that the classical Kalman filter is always in a divergent state and loses filtering function because the system equation is inaccurate. However, the LMKF and LMKF with exponential fading factor suppress filter divergence. Fig. 6 shows that LMKF must adopt classical Kalman filter before the measurement time reaches the memory length, and the error is relatively large. However, the LMKF with exponential fading factor has attenuated memory characteristic with the addition of exponential fading factor based on posteriori information, fluctuating within the interval of $[-0.5 \mathrm{~m}, 0.5 \mathrm{~m}]$, centering on error 0 in the whole interval. In addition, the error brought by the inaccuracy of the system model is reduced. Fig. 6 also shows the long-term stability of LMKF with exponential fading factor. The positive error of classical Kalman filter diverges to approximately $150 \mathrm{~m}$ when the simulation time is $600 \mathrm{~s}$, but LMKF with exponential fading factor is still basically within the interval [- $0.5 \mathrm{~m}, 0.5 \mathrm{~m}]$, guaranteeing the unbiased estimated value.

The simulation results obtained by the method in Simulation 2 in 3.2.2 are shown in Fig. 7 and 8.

Fig. 7 is a comprehensive simulation diagram of the tracking of vector motion on a $2 \mathrm{D}$ plane using the LMKF method with exponential fading factor. Fig. 8 shows the tracking errors and standard deviations of the 2D planar motion. As shown in Fig. 7 and 8, the LMKF method with exponential fading factor has a favorable tracking effect on the dynamic target under high-speed maneuvering and can inhibit the influence of noise in the environment. Thus, the filter curve can be closer to the actual motion track. 

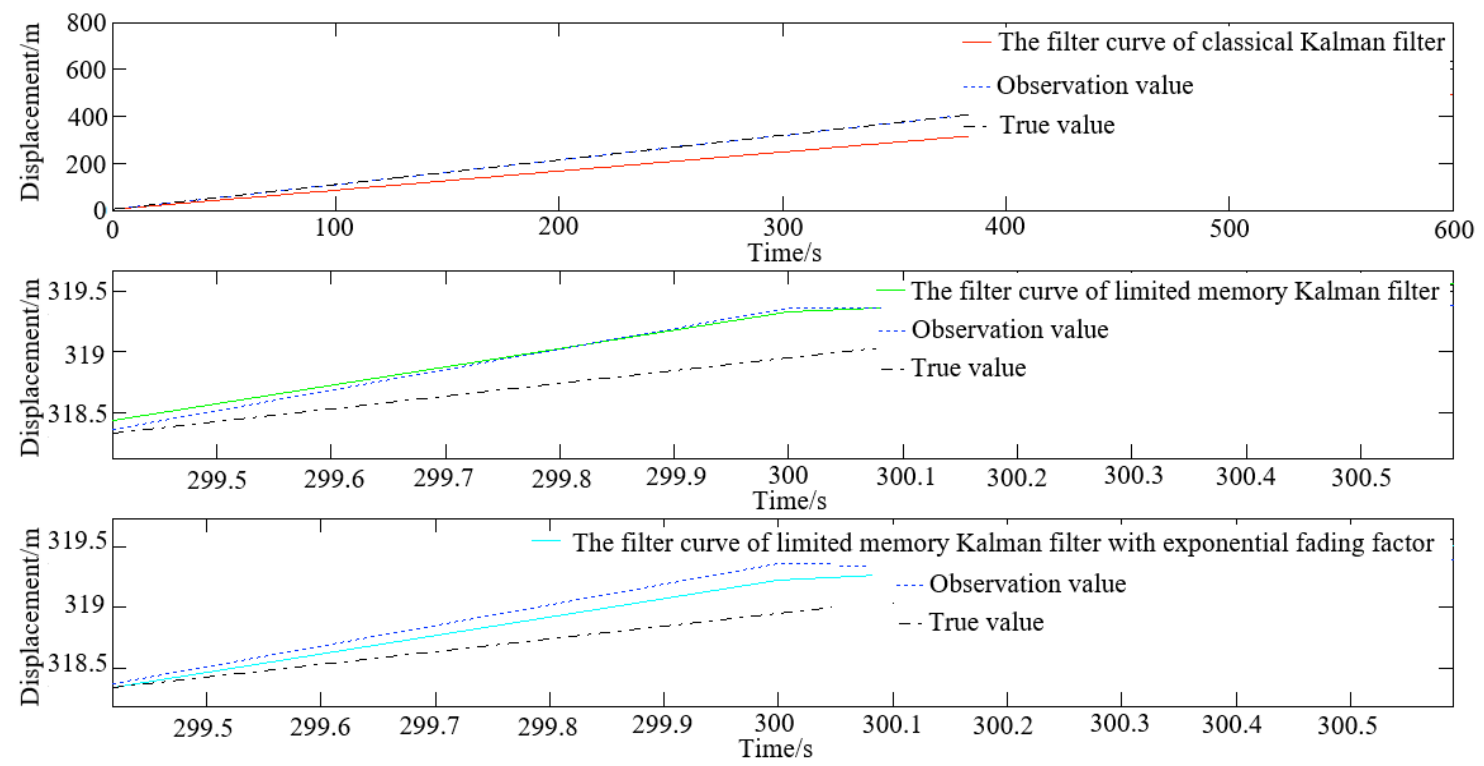

Fig. 5. Filter curves under simulation time of $600 \mathrm{~s}$ and memory length of $\mathrm{N}=50$
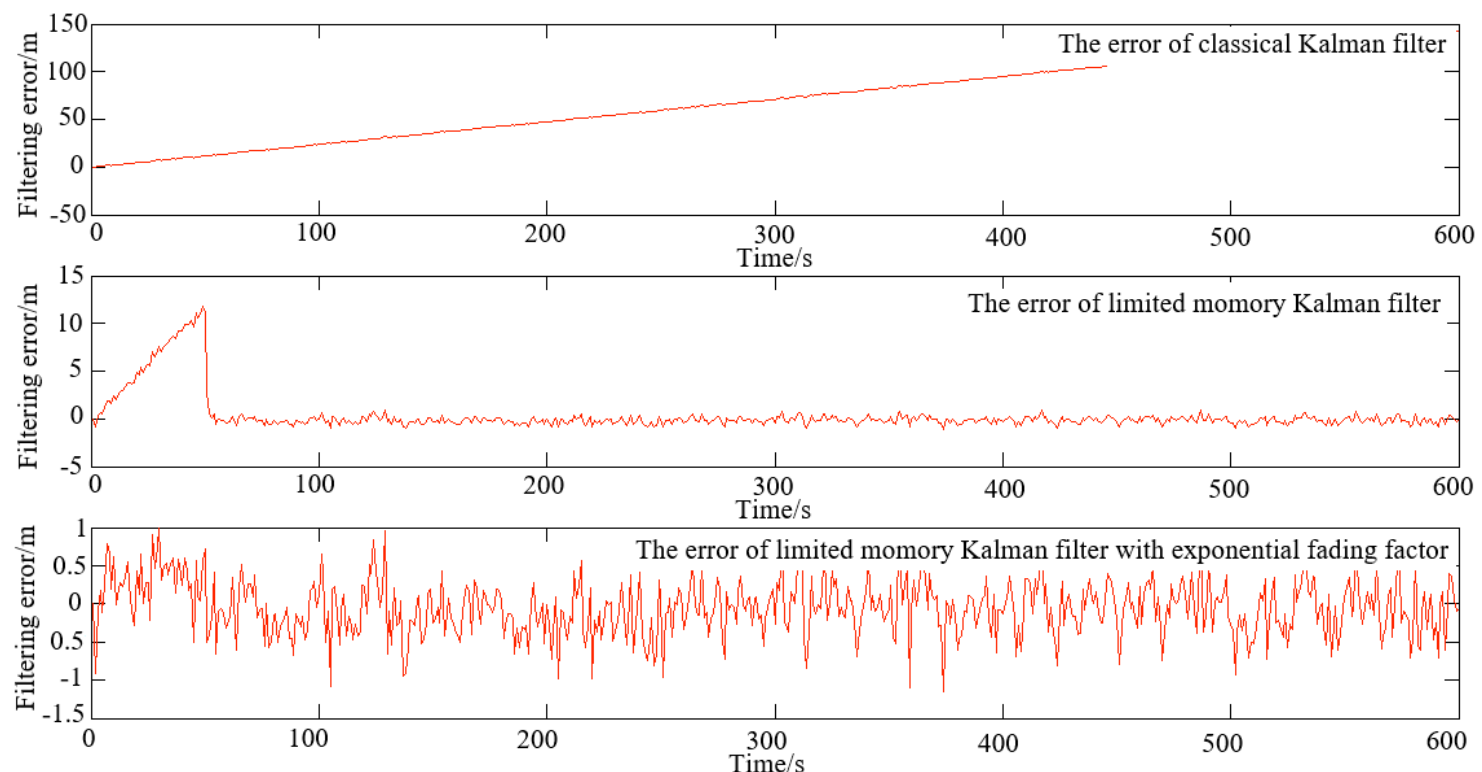

Fig. 6. Error curves under simulation time of $600 \mathrm{~s}$ and memory length of $\mathrm{N}=50$

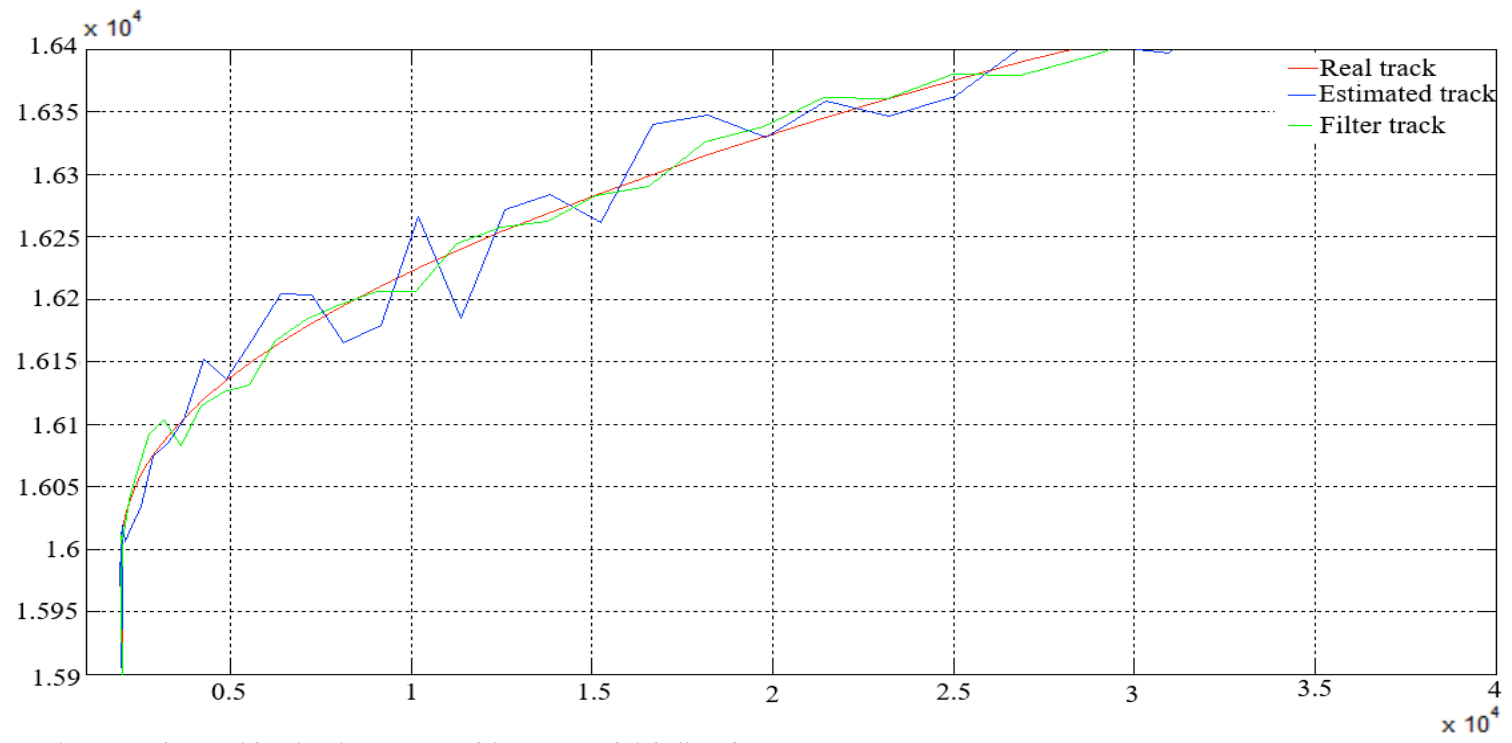

Fig.7. 2D planar motiontracking by the LMKF with exponential fading factor 

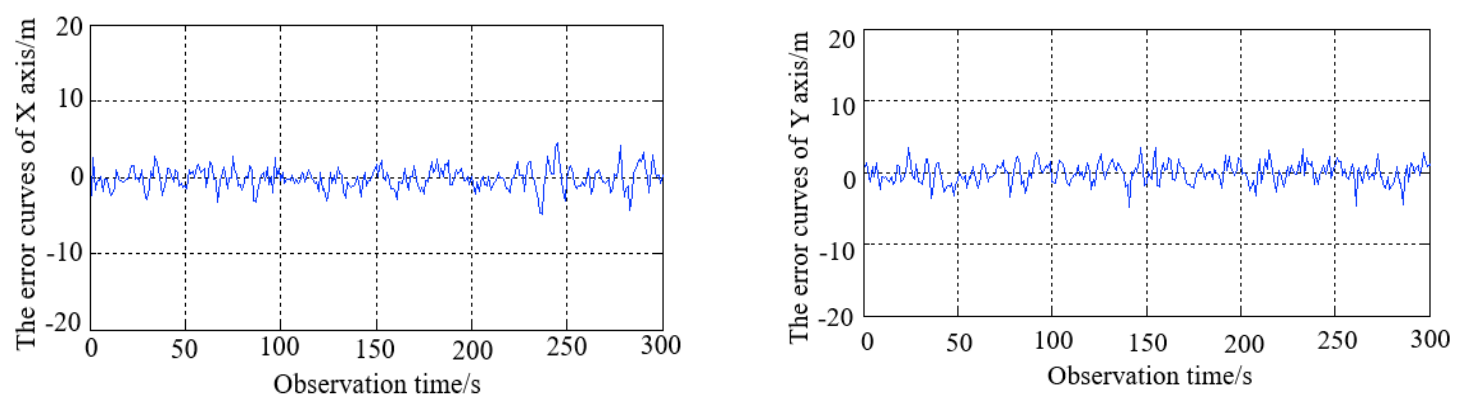

(a) Error curves for $\mathrm{X}$ and $\mathrm{Y}$ axes
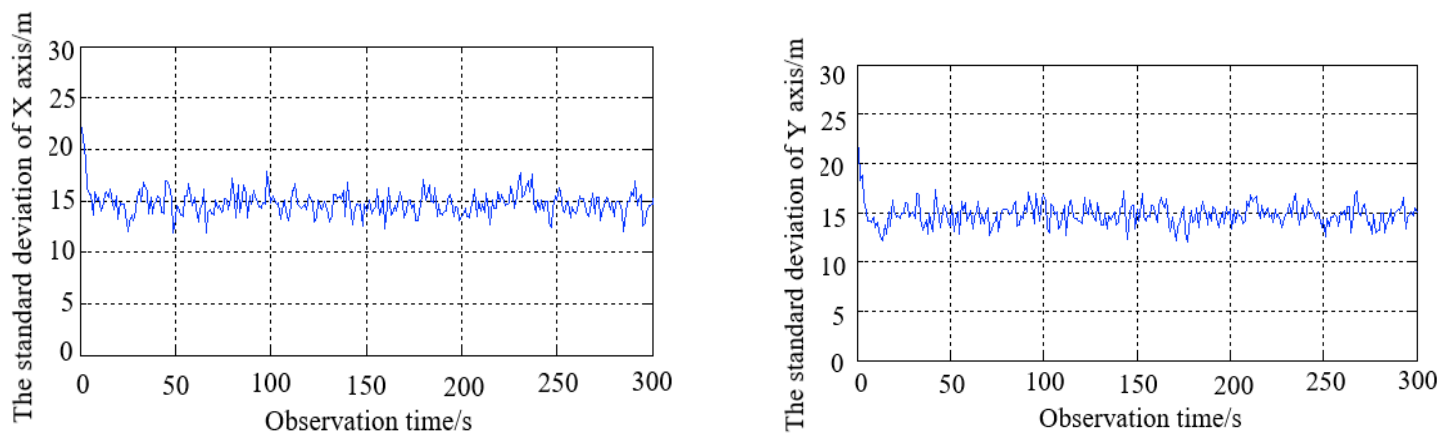

(b) Filter standard deviation curves for $\mathrm{X}$ and $\mathrm{Y}$ axes

Fig. 8. Error and standard deviation curves for tracking 2D planar motion

\subsection{Result analysis of track test with multi-sensor information fusion}

Using the test method of 3.3.3, three track curves are obtained, as shown in Fig. 9. The red line track in Fig. 9 shows that the segments $a-b l, b 1-c l$, and $g l-h l$ are close to straight line with a high track reckoning accuracy when the track is a straight line. However, due to the sudden change of robot heading, the attitude angle detected by the acceleration sensor is affected, and a large deviation occurs between the reckoning and the actual coordinates, which is reflected in segments $d 1-e l$ and $e 1-f 1$. Similar to the interference of the robot in an unknown environment, some electromagnetic disturbances in the experimental environment affect the measurement of the heading by the electronic compass. The heading measured by the electronic compass includes random noises, which are especially evident in the segment fl-gl. The error of the coded disc and odometer continues to accumulate. If the driving distance is further away, the

divergence continues to increase, and the reference significance of DR localization is lost to a certain degree. The green line tracks in Fig. 9 show that the tracks reckoned by the LMKF method with exponential fading factor proposed in study have global reliability, and the error of segments $d 2-e 2, e 2-f 2$, and $f 2-g 2$ is smaller than that of the segments $d 1-e 1, e 1-f 1$, and $f 1-g l$.

The localization error curves obtained based on odometer dead reckoning and multi-sensor information fusion using the test method stated in 3.3.3 are shown in Fig. 10. Fig. 10 shows that the maximum value of the localization error after the fusion of filtered information is reduced by $42.5 \%$ compared with the maximum error value based on odometer dead reckoning. The large random error generated by the electronic compass is filtered out, the cumulative errors of the gyroscope and the odometer are reduced, and the pose of the robot is accurately estimated.

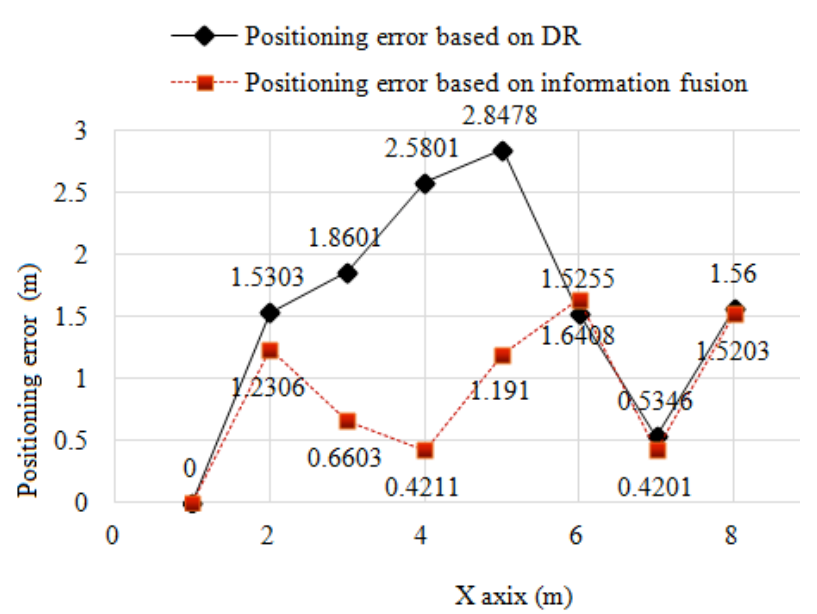

$\mathrm{X} \operatorname{axix}(\mathrm{m})$ 10

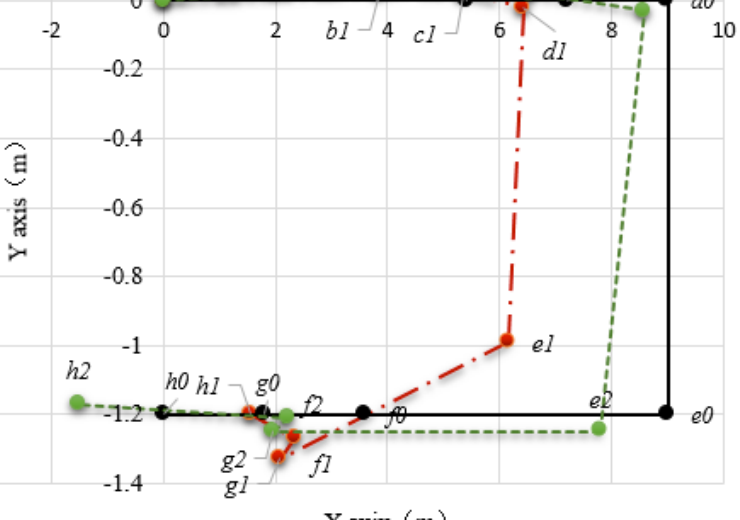

$\mathrm{X} \operatorname{axix}(\mathrm{m})$

Fig. 9 Actual track and reckoning track curves

Fig. 10. Localization error curves based on DR and multi-sensor information fusion 


\section{Conclusions}

To reduce the cumulative error of the odometer, the selflocalization method of the mobile robot is explored based on multi-sensor information fusion, and the influence of the LMKF with exponential fading factor on dead reckoning is revealed. The self-localization system of mobile robot was established, the numerical simulation and experimental studies were combined to analyze the LMKF with exponential fading factor and the dead reckoning method based on this filter, and the following conclusions could be drawn:

(1) The exponential fading factor is introduced to increase the weight of the measured value close to the current moment. Necessary correction to gain matrixes $K_{k}^{*}$ and $\bar{K}_{k}^{*}$ are guaranteed in each recurrence step. The LMKF with exponential fading factor has long-term stability and positively affects the tracking of high-speed maneuvering dynamic targets to better suppress the influence of noise in the environment and realize unbiased estimator.

(2) The LMKF with exponential fading factor can filter out the random errors generated by the electronic compass, the cumulative errors of the gyroscope and the odometer are reduced, the minimum control to the odometer error is realized, and the robot pose is accurately estimated.

Indoor experiment and theoretical study were combined, and a new LMKF method with exponential fading factor was proposed. Then, a dead reckoning algorithm of multi-sensor information fusion was obtained, which provids a certain reference for self-localization of the mobile robot. However, due to the lack of essential on-site monitoring means, this study did not consider dynamic noise monitoring data. For future study, dynamic noise monitoring data will be combined with this model and corrected which will be contributed to a more accurate recognition of the robot localization laws in a complicated environment.

\section{Acknowledgements}

This work was supported by the National Natural Science Foundation of China (Grant No. 51475148).

This is an Open Access article distributed under the terms of the Creative Commons Attribution License

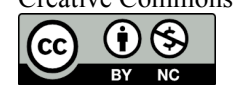

\section{References}

1. Almasri M., Elleithy K., Alajlan A., "Sensor Fusion Based Model for Collision Free Mobile Robot Navigation”. Sensors, 16(1), 2015, pp. $1-24$.

2. Liu Z. X., Xie C. X., Xie M., et al., "Mobile robot localization method based on multi-sensor information fusion laser SLAM" [EB/OL]. Retrieved from https://doi.org/10.1007/s10586-018-24747, 2018-05-13/2018-12-18

3 Klanä Ar G., Tesliä L., Krjanc I. Å., "Mobile-robot pose estimation and environment mapping using an extended Kalman filter[J]. International Journal of Systems Science, 45(12), 2014, pp. 26032618.

4 Alatise M. B., Hancke G. P., "Pose Estimation of a Mobile Robot Based on Fusion of IMU Data and Vision Data Using an Extended Kalman Filter". Sensors, 17(10), 2017, pp. 2164-2186.

5 Simanek J., Kubelka V., Reinstein M., "Improving multi-modal data fusion by anomaly detection". Autonomous Robots, 39(2), 2015, pp. $139-154$.

6 Lee J., Lim J., Lee J., "Compensated heading angles for Outdoor Mobile Robots in Magnetically Disturbed Environments". IEEE Transactions on Industrial Electronics, 65(2), 2018, pp. 1408-1419.

7 Jiang Z., Zhou W., Li H., et al., "A New Kind of Accurate Calibration Method for Robotic Kinematic Parameters Based on Extended Kalman and Particle Filter Algorithm". IEEE Transactions on Industrial Electronics, 65(4), 2018, pp. 3337-3345.

$8 \mathrm{Yu} \mathrm{H.,} \mathrm{Wang} \mathrm{J.,} \mathrm{Wang} \mathrm{B.,} \mathrm{et} \mathrm{al.,} \mathrm{"Generalized} \mathrm{total} \mathrm{Kalman} \mathrm{filter}$ algorithm of nonlinear dynamic errors-in-variables model with application on indoor mobile robot positioning". Acta Geodaetica et Geophysica, 53(1), 2018, pp.107-123.

9 Gualda D., Urena J U., Garcia-Nunez E., "Partially Constrained Extended Kalman Filter for Navigation Including Mapping Information". IEEE Sensors Journal, 16(24), 2016, pp. 9036-9046.

10 Qian J., Zi B., Wang D., et al., "The Design and Development of an Omni-Directional Mobile Robot Oriented to an Intelligent Manufacturing System”. Sensors, 17(9), 2017, pp. 2073-2088.

11 Pak J. M., Ahn C. K., Shi P., et al., "Self-recovering extended Kalman filtering algorithm based on model-based diagnosis and resetting using an assisting FIR filter". Neurocomputing, 173, 2016, pp. 645-658.

12 Luo Y., "A Technical Study of Wheel-type Mobile Robot Localization and Navigation based on Multi-information Fusion". Master thesis of Beijing Institute of Technology, China, 2011, pp. 16-39.
13 Zhang T. M., Li H. H., etc., “Agricultural Car Path Tracking and Navigation System based on Multi-source Sensor Information Fusion". Journal of Agricultural Machinery, 03 (46), 2015, pp. 3742.

14 Zhang K. Y., Liu P. L., etc., "Design and Implementation of the Indoor Robot Localization System under Multisensor Fusion". Information Technology, 11(21), 2014, pp. 83-87.

15 Li L. L., Song Z. Z., "Synchronous Mobile Robot Localization Study based on the Improved Particle Filter Algorith". Computer Measurement and Control, 21 (012), 2013, pp.3329-3332.

16 Mao L., Li Z. B., et al., "Mobile Robot Localization Study based on IR Sensors". Transducer and Microsystem Technologies, 33(12), 2014, pp. 38-41.

17 Wang Y., "Technical Study of Mobile Robot Localization based on Multi-information Fusion". Master thesis of Xidian University, China, 2015, pp. 41-47.

18 Qin T. B., "Mobile Robot Localization Study based on Multisensor Information Fusion". PLC \& FA, 11 (11), 2010, pp. 107-110.

19 Xie M., "Mobile Robot Localization Study based on Multisensor Information Fusion". Master thesis of Taiyuan University of Science and Technology, China, 2015, pp. 17-48.

20 Gao W., Li J. C., Ben Y. Y., Yang X. L., “An Adaptive Kalman Filter based on Multiple Fading Factor". Systems Engineering and Electronics, 7 (36), 2014. pp. 1405-1409.

21 Xue H. J., Guo X. S., Zhou Z. F., "SINS Initial Alignment Method based on Adaptive Multiple Fading Factors". Systems Engineering and Electronics, 3 (39), 2017, pp.620-625.

22 Katalin György, András Kelemen, László Dávid, "Unscented Kalman Filters and Particle Filter Methods for Nonlinear State Estimation". Procedia Technology, 12(1), 2014, pp. 65-74.

23 Levent Ozbek, Aliev Fazil A., "Coment on adaptive fading kalman filter with an application”. Automatica, 34 (12), 1998, pp. 16631664

$24 \mathrm{Yu}$ T.Y., Wu C.M., et al., "Application of adaptive $\alpha-\beta-\gamma$ and $\alpha-\beta-\gamma$ $\delta$ filters to tracking systems". Sensors \& materials, 29(4), 2017, pp. 419-427.

25 Safari S., Shabani F., Dan S., "Multirate multisensor data fusion for linear systems using Kalman filters and a neural network". Aerospace Science \& Technology, 39, 2014, pp. 465-471. 